\title{
Concomitant visceral and localized cutaneous leishmaniasis in two Moroccan infants
}

Tarik Mouttaki ${ }^{1,2}$, Hasnaa Maksouri ${ }^{1,2}$, Jilali El Mabrouki ${ }^{3}$, Gema Merino-Espinosa ${ }^{4}$, Hassan Fellah², Mohamed Itri ${ }^{5}$, Joaquina Martin-Sanchez ${ }^{4}$, Maha Soussi-Abdallaoui ${ }^{3,6}$, Soumiya Chiheb ${ }^{2,7}$ and Myriam Riyad ${ }^{2,6^{*}}$ (i)

\begin{abstract}
Background: Leishmaniases are vector-borne diseases caused by the protozoa of the Leishmania genus. The clinical spectrum of these diseases extends from benign dermal lesions to visceral forms. In the Mediterranean region, zoonotic visceral leishmaniasis (ZVL) is caused by L. infantum. If untreated within two years, the disease usually leads to death. In Morocco, ZVL is endemic in the north, with a hundred cases notified each year, mostly in children aged below five years. Here, we report on two clinical observations in infants presenting unusual concomitant $\mathrm{VL}$ and cutaneous leishmaniasis (CL) in Morocco.

Case presentation: In this case study, we report on two infants aged nine and 12 months old. They both have a history of febrile splenomegaly, anemia, and pallor of mucous membranes. Visceral leishmaniasis was confirmed by parasitological diagnosis (positive bone marrow smear and screening of anti-L. infantum antibodies). However, the clinical examination also showed cutaneous lesions that suggested the presence of $C L$. This was reinforced by the patients having a history of living or traveling to endemic foci. Thus, direct examination, culture, and PCR-RFLP (ITS1Hae 3) were carried out on the patients' dermal exudates. In one of the infants, CL was associated with L. infantum, while in the other it was associated with L. tropica. The infants were treated as according to the recommendations of the Ministry of Health. Both patients were cured in two months; defervescence, reduction of splenomegaly, and healing of cutaneous lesions were all observed.
\end{abstract}

Conclusions: These singular patients illustrate the clinical polymorphism of $\mathrm{CL}$ and the necessity of updating the differential diagnosis of leukemia-like syndromes, including $\mathrm{VL}$, in children living in or travelling to known endemic areas. These observations suggest a change in the Mediterranean VL phenotype that may be associated with $\mathrm{CL}$.

Keywords: Visceral leishmaniasis, Cutaneous leishmaniasis, Leishmania infantum, Leishmania tropica, Infants, Morocco

\footnotetext{
* Correspondence: myriamriyad@gmail.com

${ }^{2}$ Research Team on Immunopathology of Infectious and Systemic Diseases,

Faculty of Medicine and Pharmacy, Hassan II University of Casablanca,

Casablanca, Morocco

${ }^{6}$ Laboratory of Parasitology, Faculty of Medicine and Pharmacy, Hassan II

University of Casablanca, 19 rue Tarik Ibn Ziad, BP. 9154 Casablanca, Morocco

Full list of author information is available at the end of the article
} 


\section{Multilingual abstracts}

Please see Additional file 1 for translation of the abstract into the five official working languages of the United Nations.

\section{Background}

Leishmaniases are vector-borne parasitic diseases caused by the flagellated protozoa of the Leishmania genus. They are prevalent in 98 countries and three territories on five continents [1]. The spectrum of these diseases extends from benign cutaneous lesions that heal spontaneously to visceral forms [2]. Visceral leishmaniasis (VL) is a fatal systemic disease, if left untreated. It is caused by the Leishmania (L.) donovani complex in East Africa and the Indian subcontinent and by L. infantum in Europe, North Africa, and Latin America [3].

Zoonotic VL due to $L$. infantum is endemic in the Mediterranean region [4]. If untreated within two years, the disease usually leads to death, with approximately 300000 new VL cases occurring each year globally [1]. In Morocco, the first case of VL was described in 1922 and in 2014, 86 VL cases were notified to the Ministry of Health $[5,6]$. The disease is endemic in the north of the country, with a hundred cases reported per year, mostly in children [6-8]. The disease is more threatening in children than in adults due to the relative immaturity of a child's immune system, and is lethal if untreated [9].

In Morocco, cutaneous lesions due to Leishmania have never been reported in a patient with zoonotic VL. In contrast, $10-20 \%$ of patients develop cutaneous lesions (Post Kala azar Dermal Leishmaniasis) as a sequel of kala azar due to L. donovani $[7,10]$.

In this case study, we report on two retrospective pediatric observations of unusual clinical presentations with concurrent VL and cutaneous leishmaniasis (CL).

\section{Case presentation}

This retrospective analysis concerned two pediatric patients diagnosed in 2012. Demographic data (age, sex, place of residence, occupation for adults), diagnosis, and clinical parameters were collected through a standardized information sheet.

\section{Patient 1}

A nine-month-old girl was admitted to the Children's Hospital (University Hospital Ibn Rochd of Casablanca) with a seven-month history of febrile hepatosplenomegaly $\left(39{ }^{\circ} \mathrm{C}\right)$, anemia, pallor of mucous membranes, and pancytopenia. She was living in Casablanca but her parents reported a one-month stay in a northern endemic area of VL the previous summer.

The parasitological diagnosis (microscopic examination of bone marrow aspirate) showed the presence of Leishmania amastigotes. The screening for antibodies against
L. infantum (Leishmania IFA IgG, Vircell, Granada, Spain) was also positive (title: $1 / 320$; cut-off value: $1 / 40$ ) (see Table 1).

Furthermore, the clinical examination showed two cutaneous lesions of $5 \mathrm{~mm}$ diameter: one evolutive erythematous papule on the face and one scar lesion on the arm. The aspect of these lesions and the history of travel in a known endemic area of CL due to $L$. infantum or $L$. tropica suggested CL lesions. To confirm this hypothesis, dermic exudate was sampled from the active lesion. The microscopic examination of the corresponding stained smear was negative (absence of Leishmania amastigotes), as was the culture on Novy - Mc Neal - Nicolle (NNN) medium (absence of Leishmania promastigotes after one month). The genotyping by polymerase chain reactionrestriction fragment length polymorphism (PCR-RFLP) (ITS1 - Hae 3) carried out directly on the dermal exudate according to Schönian et al. (2003) and Mouttaki et al. (2014) protocols showed L. infantum $[11,12]$ (see Table 1).

Meglumine antimoniate (Glucantime ${ }^{\odot}$ ) was administered intramuscularly at the recommended dosage by the Ministry of Health, i.e. $20 \mathrm{mg}$ of pentavalent antimony (Sb5+) per kg per day for 20 days [7]. The patient was cured in two months; defervescence, reduction of the spleen size, hematological restoration, negativation of the parasitological control on bone marrow, and healing of cutaneous lesions were all observed.

\section{Patient 2}

A one-year-old girl was admitted to the Children's Hospital (University Hospital Ibn Rochd of Casablanca) with an impaired general condition and a three-month history of febrile $\left(39^{\circ} \mathrm{C}\right)$ hepatosplenomegaly, anemia, and pallor of mucous membranes. She was from an endemic area of both VL due to $L$. infantum and CL due to $L$. tropica (center of Morocco).

The bone marrow sample tested positive for Leishmania amastigotes. Screening utilizing the indirect fluorescent antibody technique (IFAT) for antibodies against L. infantum

Table 1 Results of the biological examinations carried out on patients 1 and 2

\begin{tabular}{lll}
\hline Biological examinations & Patient 1 & Patient 2 \\
\hline VL diagnosis & & \\
- Bone marrow stained smear & + & + \\
- Anti-L. infantum antibodies (IFAT) & $+(1 / 320)$ & $+(1 / 80)$ \\
- Anti-L. infantum antibodies (WB) & $\mathrm{ND}$ & $+(14-16 \mathrm{kD})$ \\
CL diagnosis & & \\
- Skin stained smear & - & + \\
- Culture (NNN) & - & + \\
- PCR-RFLP & L. infantum & L. tropica \\
\hline
\end{tabular}

+: Positive; -: Negative; ND: Not done

${ }^{a}$ Cut-off value: $1 / 40$ 
(Leishmania IFA IgG, Vircell, Granada, Spain) was weakly positive (title: $1 / 80$; cut-off value: $1 / 40$ ). This latter low value and the altered general status led us to carry out a specific western blot (WB) screening for anti-L. infantum antibodies (LEISHMANIA Western Blot Ig G, LDBIO Diagnostics, Lyon, France) and a screening for anti-HIV antibodies. Only the $L$. infantum serology by WB was positive (antibodies anti-14-16 kD proteins) (see Table 1).

Moreover, the clinical examination revealed two cutaneous papule lesions evolving for three months on the face, which did not heal with a previously prescribed dermal antibiotherapy before the patient's hospital admission. These lesions as well as the infant's origin from a known endemic area of CL due to L. infantum and/or L. tropica led us to sample the dermal exudate. The corresponding stained smear was positive as was the culture on NNN medium. The genotyping by PCR-RFLP carried out on the dermal sample and the isolate revealed L. tropica.

Meglumine antimoniate (Glucantime ${ }^{\circ}$ ) was administered following the same protocol as for patient 1, but for 30 days. The patient was cured after two months of treatment; defervescence, reduction of splenomegaly, and healing of cutaneous lesions were all observed.

\section{Discussion and conclusions}

In South Europe and North Africa, the Mediterranean type of VL is usually caused by L. infantum, and infants or children aged up to four years are frequently affected. The incubation period ranges from 10 days to over one year, and the disease onset is usually gradual. The common symptoms are fever, malaise, shivering or chills, weight loss, anorexia, and discomfort in the left hypochondrium. The common clinical signs are non-tender splenomegaly, with or without hepatomegaly, wasting and pallor of mucous membranes. Signs of malnutrition (edema, skin and hair changes) develop as the disease progresses. Intercurrent infections are common [13].

Clinically, both infants observed in this study presented clinical signs suggestive of VL. This hypothesis was reinforced by the fact that they either lived or stayed in known endemic VL foci according to Ministry of Health data. The positive bone marrow smear and the anti-L. infantum serology allowed us to confirm VL in both cases. These children were then treated with Glucantime ${ }^{\bullet}$ and were cured within two months. In Morocco, the recommended treatment for $\mathrm{VL}$ is antimonials $(20 \mathrm{mg} \mathrm{Sb}+/ \mathrm{kg} /$ day for three weeks), and the cure rate is $99 \%[7,14]$.

Furthermore, both infants presented signs suggestive of $\mathrm{CL}$, as follows: cutaneous lesions on exposed skin surfaces (face and arm), history of resistance to dermal antibiotic treatment, and history of living or traveling the previous summer to known endemic foci of CL due to $L$. tropica or $L$. infantum. While the conventional parasitological diagnostic methods (dermal smears and culture) confirmed the diagnosis only for patient 2 , the genotyping on the dermal samples revealed $L$. infantum in patient 1 and $L$. tropica in patient 2.

In Morocco, VL is a zoonotic rural disease endemic in northern regions and cases are sporadically reported in some southern regions. Leishmania infantum is also responsible for sporadic CL in North Africa in the same geographical area as VL [15]. In Morocco, the first $L$. infantum CL patient was reported in 1996 from an active focus of canine VL in the northern Rif mountains [16]. In fact, most CL cases due to L. infantum are notified from one focus (Sidi Kacem in the northwest), where sporadic cutaneous forms have been described as two-year-course single lesions [17-19]. To our knowledge, the association of $\mathrm{VL}$ and $\mathrm{CL}$ in the same human immunocompetent host has not been previously described.

Leishmania tropica is responsible for anthroponotic CL in Morocco. In the 1980s, the disease was hypoendemic in center-south rural foci. In the 1990s, urban epidemic foci appeared in the north [20, 21]. In fact, CL due to L. tropica has the widest geographic distribution in the country and the highest incidence in North Africa $[8,15,22]$.

The CL cases presented in this study are interesting due to their association with a visceral syndrome. Until now, the clinical definition of VL in Morocco by the Ministry of Health highlighted the absence of cutaneous lesions. Thus, $L$. infantum could be responsible for both the visceral and cutaneous symptomatology in patient 1 , whereas two different species, L. infantum and L. tropica, could be respectively responsible for the visceral and cutaneous symptomatology in patient 2.

For patient 1 (CL due to L. infantum), the symptomatology could be explained either by the inoculation of different viscerotropic and dermotropic strains of $L$. infantum through the vector bite, or by the immunological status of children, which would have allowed the visceral dissemination of a dermotropic strain of $L$. infantum. Patient 2, who had CL due to L. tropica, lived in a co-endemic region of VL due to L. infantum and CL due to $L$. tropica. We could not discriminate between $L$. tropica and $L$. infantum on the bone marrow. We therefore cannot state with certainty that this patient's VL was due to L. infantum. Few cases of VL caused by L. tropica have been reported in India, Iran, and Israel [10, 13, 23].

Furthermore, IFAT serology showed a low antibody title $(1 / 80)$ close to the cut-off value in patient 2 , which is consistent with a cross-reaction. Cross-reactions have also been described with $L$. tropica using WB [24]. If the VL of this patient was caused by L. infantum, this would highlight the possibility of a coinfection by two endemic species in the same focus. It is usually considered that vector and human hosts are infected by a single species and that the natural infection by one Leishmania species would protect against reinfections by homologous or 
heterologous species $[25,26]$. However, natural human coinfections have been reported in Bolivia, Brazil, Iran, Iraq, Mexico, and Peru [25, 27]. In Morocco, these observations must be kept in mind due to the increasing number of reported co-endemic CL foci due to $L$. infantum and L. tropica, or L. tropica and L. major $[8,17]$.

Finally, these patients illustrate the clinical polymorphism of $\mathrm{CL}$ and its difficult clinical diagnosis, even in endemic regions. They also highlight the importance of updating the differential diagnosis of leukemia-like syndromes, including VL in infants living in or travelling to known endemic areas. Indeed the patients staying during the summer in known foci was a pivotal anamnestic fact that directed the clinical diagnosis towards VL and then $\mathrm{CL}$ that were confirmed by the biological examinations. Our observations also suggest a change in the Mediterranean VL phenotype that may be associated to cutaneous lesions. It is thus important to characterize Leishmania species in any unusual clinical situation and to report particular cases such as coinfections in order to contribute to better epidemiological and physiopathological knowledge of leishmaniases in endemic areas.

\section{Additional file}

Additional file 1: Multilingual abstracts in the five official working languages of the United Nations. (PDF $346 \mathrm{~kb}$ )

\section{Abbreviations}

CL: Cutaneous leishmaniasis; IFAT: Indirect fluorescent antibody technique; PCR-RFLP: Polymerase chain reaction-restriction fragment length polymorphism; VL: Visceral leishmaniasis; WB: Western blot; ZVL: Zoonotic visceral leishmaniasis

\section{Acknowledgments}

Not applicable.

\section{Funding}

Not applicable.

\section{Availability of data and materials}

All the data of patients 1 and 2 are presented in § Case Presentation and Table 1 . The authors are ready to provide any supplementary information upon request.

\section{Authors' contributions}

MR and SC jointly coordinated the study, with contributions from JM-S, MS$A$, and HF. TM and HM performed the culture analysis, extracted the DNA from the samples, and undertook the PCR-RFLP. GM-E retested and controlled the molecular assays. JEM performed the direct examination and IFAT. $\mathrm{Ml}$ was in charge of managing the patients. MR and SC analyzed the data and drafted the paper, which was then revised by JM-S, MS-A, and HF. All authors read and approved the final paper for publication.

\section{Ethics approval and consent to participate}

This work was conducted according to the principles specified in the Declaration of Helsinki and under the local ethical guidelines (Ethics Committee for Biomedical Research, Faculty of Medicine and Pharmacy, Hassan II University of Casablanca, Morocco). The retrospective patients' data were anonymized. Parents gave their consent to publish the data about their children.

\section{Consent for publication}

Not applicable.

\section{Competing interests}

The authors declare that they have no competing interests.

\section{Author details}

${ }^{1}$ Centre of Doctoral Studies on Health Sciences (CED. des Sciences de la Santé), Doctoral School of Immunopathology, Faculty of Medicine and Pharmacy, Hassan II University of Casablanca, Casablanca, Morocco. ${ }^{2}$ Research Team on Immunopathology of Infectious and Systemic Diseases, Faculty of Medicine and Pharmacy, Hassan II University of Casablanca, Casablanca, Morocco. ${ }^{3}$ Laboratory of Parasitology and Mycology, University Hospital Ibn Rochd of Casablanca, Casablanca, Morocco. ${ }^{4}$ Department of Parasitology, Faculty of Pharmacy, University of Granada, Granada, Spain. ${ }^{5}$ Children's Hospital, University Hospital Ibn Rochd of Casablanca, Casablanca, Morocco. ${ }^{6}$ Laboratory of Parasitology, Faculty of Medicine and Pharmacy, Hassan II University of Casablanca, 19 rue Tarik Ibn Ziad, BP. 9154 Casablanca, Morocco. ${ }^{7}$ Department of Dermatology, University Hospital Ibn Rochd of Casablanca, Casablanca, Morocco.

Received: 21 June 2017 Accepted: 27 March 2018

Published online: 12 April 2018

\section{References}

1. World Health Organization, Department of control of neglected tropical diseases. Sustaining the drive to overcome the global impact of neglected tropical diseases. In: Second WHO report on neglected tropical diseases. WHO/HTM/NTD/2013.1.

2. Salam N, Al-Shaqha WM, Azzi A. Leishmaniasis in the Middle East: incidence and epidemiology. PLoS Negl Trop Dis. 2014;8(10):e3208.

3. Tasew G, Gadisa E, Abera A, Zewude A, Chanyalew M, Aseffa A, et al. In vitro permissiveness of bovine neutrophils and monocyte derived macrophages to Leishmania donovani of Ethiopian isolate. Parasit Vectors. 2016;9:218.

4. Postigo JAR. Leishmaniasis in the World Health Organization eastern Mediterranean region. Int J Antimicrob Agents. 2010. https://doi.org/10. 1016/j.jantimicag.2010.06.023.

5. Klippel S, Monier-Vignard F. Premier cas de Kala-azar d'origine marocaine. Soc Med des Hospitaux de Paris. 1922;20:72-85. (in French)

6. Ministry of Health, Kingdom of Morocco, Direction de la Planification et des Ressources Financières, édition 2015. Santé en chiffres; 2014. http://www. sante.gov.ma/Publications/Etudes_enquete/Pages/default.aspx. (in French).

7. Ministry of Health, Kingdom of Morocco, Direction de l'Epidémiologie et de la Lutte contre les Maladies. Lutte contre les Leishmanioses, guide des activités; 2010.

8. Ministry of Health, Kingdom of Morocco, Direction de l'Epidémiologie et de la Lutte contre les Maladies, Service des Maladies Parasitaires. Bilan des activités des programmes de lutte contre les maladies parasitaires, année 2010; 2010. (in French).

9. Cascio A, Colomba C. Childhood Mediterranean visceral leishmaniasis. Infez Med. 2003;11(1):5-10.

10. Khanra S, Bandopadhyay SK, Chakraborty P, Datta S, Mondal D, Chatterjee $M$, et al. Characterization of the recent clinical isolates of Indian kala-azar patients by RAPD-PCR method. J Parasit Dis. 2011;35(2):116-22.

11. Schönian G, Nasereddin A, Dinse N, Schweynoch C, Schallig HD, Presber W, et al. PCR diagnosis and characterization of Leishmania in local and imported clinical samples. Diagn Microbiol Infect Dis. 2003;47(1):349-58.

12. Mouttaki T, Morales-Yuste M, Merino-Espinosa G, Chiheb S, Fellah H, MartinSanchez J, et al. Molecular diagnosis of cutaneous leishmaniasis and identification of the causative Leishmania species in Morocco by using three PCR-based assays. Parasit Vectors. 2014;7:420.

13. World Health Organization. Control of the leishmaniases: report of a meeting of the WHO expert committee on the control of Leishmaniases. Geneva; 2010. WHO technical report series 949 2010. http://apps.who.int/ iris/bitstream/10665/44412/1/WHO_TRS_949_eng.pdf.

14. Alvar J, Vélez ID, Bern C, Herrero M, Desjeux P, Cano J, et al. WHO Leishmaniasis control team. Leishmaniasis worldwide and global estimates of its incidence. PLoS One. 2012. https://doi.org/10.1371/journal.pone. 0035671.

15. Rioux JA, Gallego M, Dereure J, Perieres J, Lamrani A, Riera C, et al. Human cutaneous leishmaniasis due to Leishmania infantum zymodeme MON-24 in Morocco. Bull Soc Fr Parasitol. 1996:14:179-83.

16. Rhajaoui M, Nasereddin A, Fellah H, Azmi K, Amarir F, Al-Jawabreh A, et al. New clinico-epidemiologic profile of cutaneous leishmaniasis, Morocco. Emerg Infect Dis. 2007. https://doi.org/10.3201/eid1309.070946. 
17. Rhajaoui M. Human leishmaniases in Morocco: a nosogeographical diversity. Pathol Biol (Paris). 2011;59(4):226-9.

18. Hakkour M, Hmamouch A, El Alem MM, Rhalem A, Amarir F, Touzani M, et al. New epidemiological aspects of visceral and cutaneous leishmaniasis in Taza, Morocco. Parasit Vectors. 2016;9(1):612.

19. Guessous-Idrissi N, Chiheb S, Hamdani A, Riyad M, Bichichi M, Hamdani S, et al. Cutaneous leishmaniasis: an emerging epidemic focus of Leishmania tropica in North Morocco. Trans R Soc Trop Med Hyg. 1997;91(6):660-3.

20. Rhajaoui M, Fellah H, Pratlong F, Dedet JP, Lyagoubi M. Leishmaniasis due to Leishmania tropica MON-102 in a new Moroccan focus. Trans R Soc Trop Med Hyg. 2004;98(5):299-301.

21. Aoun K, Bouratbine A. Cutaneous leishmaniasis in North Africa: a review. Parasite. 2014;21:14.

22. Kahime K, Boussaa S, Laamrani-El Idrissi A, Nhammi H, Boumezzough A. Epidemiological study on acute cutaneous leishmaniasis in Morocco. JAD. 2016. https://doi.org/10.1016/j.joad.2015.08.004

23. Hosseininasab A, Sharifi I, Daei MH, Zarean M, Dadkhah M. Causes of pediatric visceral leishmaniasis in southeastern Iran. Iran J Parasitol. 2014; 9(4):584-7.

24. Zeyrek FY, Korkmaz M, Ozbel Y. Serodiagnosis of anthroponotic cutaneous leishmaniasis (ACL) caused by Leishmania tropica in Sanliurfa Province, Turkey, where ACL is highly endemic. Clin Vaccine Immunol. 2007;14(11): 1409-15.

25. Veland N, Valencia BM, Alba M, Adaui V, Llanos-Cuentas A, Arevalo J, et al. Simultaneous infection with Leishmania (Viannia) braziliensis and L. (V.) lainsoni in a Peruvian patient with cutaneous leishmaniasis. Am J Trop Med Hyg. 2013;88(4):774-7.

26. Reale S, Maxia L, Vitale F, Glorioso NS, Caracappa S, Vesco G. Detection of Leishmania infantum in dogs by PCR with lymph node aspirates and blood. J Clin Microbiol. 1999;37(9):2931-5.

27. Shirian S, Oryan A, Hatam GR, Daneshbod Y. Mixed mucosal leishmaniasis infection caused by Leishmania tropica and Leishmania major. J Clin Microbiol. 2012:50(11):3805-8.

\section{Submit your next manuscript to BioMed Central and we will help you at every step:}

- We accept pre-submission inquiries

- Our selector tool helps you to find the most relevant journal

- We provide round the clock customer support

- Convenient online submission

- Thorough peer review

- Inclusion in PubMed and all major indexing services

- Maximum visibility for your research

Submit your manuscript at www.biomedcentral.com/submit 